\title{
Biochemical Systems Theory and Metabolic Control Theory: 2. The Role of Summation and Connectivity Relationships
}

\author{
MICHAEL A. SAVAGEAU, EBERHARD O. VOIT,* AND DOUGLAS H. IRVINE \\ Department of Microbiology and Immunologv, The University of Michigan, \\ Ann Arbor, Michigan 48109 \\ Recelved 11 August 1986: revised 18 June 1987
}

\begin{abstract}
Perhaps the major obstacle to recognizing the relatedness of Biochemical Systems Theory (BST) and a subsequently developed approach some have called Metabolic Control Theory (MCT) is the summation and connectivity relationships. These are the most visible and central features of the MCT approach to the understanding of intact biochemical systems, whereas in the RST approach they appear to be invisible and peripheral. Generalized versions of these relationships are shown to be inherent to BST, and it is shown how their role differs from that within MCT. The significance of summation and connectivity relationships is shown to be historical and secondary in the sense that one can understand fully the integrated behavior of complex biochemical systems in steady state with BST and never explicitly invoke these relationships. It also is shown that the summation and connectivity relationships in MCT have inherent limitations that make them inadequate as the basis for a general theory of biochemical systems. The results in this paper, together with those in the previous paper, clearly demonstrate that MCT is a special case of BST.
\end{abstract}

\section{INTRODUCTION}

Largely as a result of the spectacular advances in molecular biology during the past several decades, a complementary integrative approach to complex biochemical systems has become not only possible but absolutely necessary if we are to understand fully the integrated behavior of such systems. In the late 1960s an integrative approach [13-17] was introduced that represented the first theory to differ significantly from the two wellestablished approaches based on the Linear Formalism and the MichaelisMenten Formalism. This approach is called Biochemical Systems Theory (BST); for reviews see Savageau [18, 21-23]. In the mid 1970s another approach was developed $[8,5]$, which some have referred to as Metabolic

\footnotetext{
*Current address: Department of Biometry, Medical University of South Carolina, Charleston, SC 29425.
}

MATHEMATICAL BIOSCIENCES 86:147-169 (1987)

CElsevier Science Publishing Co., Inc., 1987

52 Vanderbilt Ave., New York, NY 10017

$0025-5564 / 87 / \$ 03.50$ 
Control Theory (MCT). For a recent review see [9]. Although MCT appears to cover the same phenomena and to yield the same results as BST, the developers of MCT have treated it as entirely different from BST, and in this regard the field has become confused with seemingly different approaches [28], which in fact are not fundamentally different and which have led to rediscoveries and repetition [31]. This unfortunate situation has developed at least in part because, until very recently, there have been no meaningful cross references between these approaches and no detailed comparisons have been published. Clarification of their relatedness is long overdue.

In the preceding paper [27] we have identified two issues that are central to such clarification: (1) the fundamental character of the formalism that underlies these two approaches, and (2) the role of "summation" and "connectivity" relationships in BST and MCT. The first issue was treated at length in the preceding paper. It was demonstrated that the same Power-Law Formalism underlies both BST and MCT, and that the variant of the Formalism used for BST makes it a more systematic and general theory than MCT.

In this paper we will show that generalized summation and connectivity relationships are inherent to BST. In fact, they will be shown to be mathematically identical to the familiar orthogonality relationships of linear systems. These generalized relationships reduce to the special cases of summation and connectivity relationships found by Kacser and Burns [8] and Heinrich and Rapoport [5] when the restrictions of MCT are taken into account. The role of summation and connectivity relationships within BST and MCT also will be discussed, and further similarities and differences between BST and MCT will be presented.

\section{STEADY-STATE SOLUTIONS}

In BST the fundamental equations that describe biochemical systems are written explicitly in terms of the underlying Power-Law Formalism [14]:

$$
d X_{i} / d t=\alpha_{i} \prod_{j=1}^{n+m} X_{j}^{g_{i j}}-\beta_{i} \prod_{j=1}^{n+m} X_{j}^{h_{i j}}, \quad i=1, \ldots, n .
$$

The $X_{i}$ are variables ( $n$ are "internal" or dependent and $m$ are "external" or independent); they typically refer to concentrations, but they also may refer to other physical or chemical quantities. The parameters $\alpha_{i}$ and $\beta_{i}$ are rate constants for the net increase (synthesis, import, concentration, etc.) and net decrease (degradation, export, dilution, etc.) of $X_{i}$, respectively. The parameters $g_{i j}$ and $h_{i j}$ are kinetic orders that also characterize these net processes.

In steady state, the time derivatives are equal to zero, and Equation (1) can be written as

$$
[A] y]=b],
$$


where

$$
\begin{aligned}
y_{i} & =\log X_{i}, \\
b_{i} & =\log \left(\beta_{i} / \alpha_{i}\right), \\
a_{i j} & =g_{i j}-h_{i j} .
\end{aligned}
$$

Equation (2) has a steady-state solution provided the system determinant is nonzero [14].

The explicit steady-state solution in symbolic form gives the dependent variables and fluxes within an arbitrary system expressed directly in terms of the external or independent variables and molecular parameters associated with the individual enzymes and processes of the system [14]:

$$
\left.\left.y]_{\text {in }}=[L] y\right]_{\mathrm{ex}}+[M] b\right]
$$

and

$$
\log V]=\log \alpha]+[G] y] .
$$

The subscripts "in" and "ex" refer to vectors of internal and external variables, $[L]$ and $[M]$ are matrices whose elements are functions only of the kinetic orders $g_{i j}$ and $h_{i j}, V_{i}$ is the net flux through the $X_{i}$ pool, $\alpha_{i}$ is the corresponding rate constant, and $[G]$ is the matrix of kinetic orders $g_{i j}$. See Savageau et al. [27] for additional discussion.

Although this solution provides a direct answer to the question of how any dependent variable or flux is determined by the independent variables and molecular parameters, it is convenient for relating elemental components and system behavior also to define particular systemic concepts.

\section{SYSTEMIC DESCRIPTIONS}

From the explicit symbolic solution in Equations (3) and (4) one can calculate systemic properties directly and can exhibit their relation to the parameters of the underlying molecular mechanisms [16, 17].

\section{I IOGARITIMIC-GAIN FACTORS}

The matrix $[L]$ in Equation (3) is an $m \times n$ array of elements that are functions only of the kinetic orders. These elements, defined as logarithmicgain factors $[17,18]$ according to well-established precedents $[2,11,29]$, are systemic properties that relate the change in the logarithm of an independent variable $\left(X_{j}\right)$ to the resulting change in the logarithm of a dependent variable $\left(X_{i}\right)$ :

$$
L_{i j}=\frac{\partial \log X_{i}}{\partial \log X_{j}}=\frac{\partial y_{i}}{\partial y_{j}}
$$


Inspection of these quantities in Equation (3) yields fundamental relationships between elemental components and system behavior: the percentage change in an external variable $\left(X_{j}\right)$ is transmitted throughout the system, and the resultant effect on any given internal variable $\left(X_{i}\right)$ is the original percentage multiplied by the systemic quantity $L_{i j}$. Hence, the solution in Equation (3) exhibits the relationship between this systemic quantity and the underlying molecular properties, the kinetic orders. For example, see Equation (37). Numerous examples of the use of such relationships also are given elsewhere (e.g., see $[18,21])$.

\subsection{SENSITIVITIES-RATE CONSTANTS}

The matrix $[M]$ in Equation (3) is an $n \times n$ array of elements that also are functions only of the kinetic orders. These elements, defined as sensitivities of dependent variables with respect to rate constants [16, 18], again according to well-established precedents $[2,29,3]$, are systemic properties that relate the change in the logarithm of an $\alpha$ or $\beta$ rate constant to the change in the logarithm of a dependent variable. For example, from Equation (3),

$$
y_{i}=\sum_{j=n+1}^{n+m} L_{i j} y_{j}+\sum_{j=1}^{n} M_{i j}\left(\log \beta_{j}-\log \alpha_{j}\right), \quad i=1, \ldots, n,
$$

and

$$
\begin{aligned}
& S\left(X_{i}, \alpha_{j}\right)=\frac{\partial \log X_{i}}{\partial \log \alpha_{j}}=-\frac{\partial y_{i}}{\partial b_{j}}, \\
& S\left(X_{i}, \beta_{j}\right)=\frac{\partial \log X_{i}}{\partial \log \beta_{j}}=\frac{\partial y_{i}}{\partial b_{j}} .
\end{aligned}
$$

Thus,

$$
S\left(X_{i}, \beta_{j}\right)=-S\left(X_{i}, \alpha_{j}\right)=M_{i j}
$$

Inspection of these quantities in Equation (3) yields another class of relationships between elemental components and system behavior: the percentage change in a rate constant parameter $\left(\alpha_{j}\right.$ or $\left.\beta_{j}\right)$ is propagated throughout the system, and the resultant effect on any given dependent variable internal to the system $\left(X_{i}\right)$ is the original percentage multiplied by the systemic property $M_{i j}$. The solution in Equation (3) exhibits the relationship between this systemic property and the underlying molecular properties, the kinetic orders. For example, see Equation (37). 


\subsection{SENSITIVITIES - KINETIC ORDERS}

The effect of variation in kinetic orders can be calculated directly from the steady-state solution in BST. The sensitivity of a dependent variable to a change in kinetic order is defined as

$$
S\left(y_{i}, g\right)=\frac{\partial y_{i}}{\partial g} \frac{g}{y_{i}},
$$

where $g$ is any given parameter $g_{i j}$ (or $h_{i j}$ ) $[17,18]$. With this definition expressions comparable to those produced with variation in rate constants can be obtained. In general,

$$
\begin{aligned}
S\left(y_{i}, g\right)= & \frac{\partial y_{i}}{\partial g} \frac{g}{y_{i}}=\frac{\sum_{j=n+1}^{n+m} \frac{\partial L_{i j}}{\partial g} \frac{g}{L_{i j}} L_{i j} y_{j}+\sum_{j=1}^{n} \frac{\partial M_{i j}}{\partial g} \frac{g}{M_{i j}} M_{i j} b_{j}}{\sum_{j=n+1}^{n+m} L_{i j} y_{j}+\sum_{j=1}^{n} M_{i j} b_{j}} \\
= & \frac{\sum_{j-n+1}^{n+m} S\left(L_{i j}, g\right) L_{i j} y_{j}+\sum_{j=1}^{n} S\left(M_{i j}, g\right) M_{i j} b_{j}}{\sum_{j=n+1}^{n+m} L_{i j} y_{j}+\sum_{j=1}^{n} M_{i j} b_{j}} .
\end{aligned}
$$

This can be viewed as a weighted average of the sensitivities of the individual coefficients $L_{i j}$ and $M_{i j}$, which can be seen more clearly when the variables $X_{i}$ are normalized with respect to their steady-state values. The vector $\left.b\right]$ is then zero, $\left.y]_{\mathrm{in}}=[L] y\right]_{\mathrm{ex}}$, and Equation (10) reduces to

$$
S\left(y_{i}, g\right)=\frac{\sum_{j=n+1}^{n+m} S\left(L_{i j}, g\right) L_{i j} y_{j}}{\sum_{j=n+1}^{n+m} L_{i j} y_{j}}
$$

Furthermore, in cases for which there is a single independent variable, or for which all $S\left(L_{i j}, g\right)$ are identical,

$$
S\left(y_{i}, g\right)=S\left(L_{i j}, g\right) .
$$

These sensitivities have been used in numerous applications of BST (e.g., see $[18,21])$. Change in a kinetic order is propagated throughout the system, and the resultant effects are observed in variables internal to the system. The relationship between elemental components and system behavior is made evident by direct calculation from Equation (3).

Any parameter change in a system is ultimately manifested by changes in the two fundamental types of parameters in the Power-Law Formalism - rate 
constants and kinetic orders. Although we can determine mathematically the consequences of a change in any of these individual parameters, physical or genetic alteration of a system generally affects several parameters simultaneously $[18 ; 21$, Chapter $9 ; 25 ; 7]$. The net change in any systemic property must therefore be determined by addition of the contributions from each of the affected parameters. We shall see examples in Section 5.

In MCT there is no explicit analytical solution corresponding to Equation (3) and no theorem, corresponding to Equations (13) or (14) in Savageau et al. [27], for the existence of such a steady state. Also the systemic properties corresponding to sensitivities with respect to kinetic orders, which are the most numerous in a system, have not been defined or utilized in MCT. The influence of independent variables on the behavior of the system has been defined in terms of "response coefficients" [8], which are the flux analogs of the logarithmic-gain factors (see Section 3.1) first defined by Savageau $[17,18]$. However, the use of logarithmic gains (response coefficients) has not been developed systematically as part of MCT. The influences, termed "concentration control coefficients" (rate-constant sensitivities), of "enzyme levels" (rate constants) on the dependent variables of the system recently have been related directly to the "elasticities" (kinetic orders) in MCT (sce [33]). These relationships, however, are not derived directly from an explicit steady-state solution corresponding to Equation (3); rather, these relationships have been derived by use of another set of relationships between sensitivities (control coefficients) and kinetic orders (elasticities) - the summation and connectivity relationships - that are the primitive equations in $\mathrm{MCT}$.

Specific summation and connectivity relationships were found by Kacser and Burns [8] and Heinrich and Rapoport [5]. These relationships were confirmed in BST [21, Chapter 9]. Westerhoff and Chen [33] have found additional relationships of a similar type in MCT. The relationships in BST are generalized summation and connectivity relationships that differ from those in MCT, as will be seen below.

\section{ORTHOGONALITY PROPERTIES}

Because of the linear structure of the system's steady-state equations (log form) in BST, the effects of change in the rate constants (or $b$ parameters) can be considered independently of those in the independent $y$ variables [Equation (3)]. For simplicity, and without loss of generality, one may examine the effects of changes in rate constants ( $b$ parameters) in an autonomous system, i.e. in a system with no independent variables. The steady-state solution for such a system then can be written

$$
y]=[M] b],
$$


where $[M]=[A]^{-1}$, and, as previously indicated,

$$
M_{i j}=S\left(X_{i}, \beta_{j}\right)=-S\left(X_{i}, \alpha_{j}\right) .
$$

Furthermore, since

$$
[M][A]=[I] \quad \text { (identity matrix), }
$$

Equation (15) may be thought of as a set of constraints given by

$$
\sum_{j=1}^{n} M_{i j} a_{j k}=\delta_{i k}
$$

where $\delta_{i k}$ is the Kronecker delta symbol equal to 1 for $i=k$ and 0 for $i \neq k$. These are the familiar orthogonality properties of linear systems (e.g., see [1]).

The generalized summation and connectivity relationships are mathematically identical to these two properties [Equation (14) and Equation (16)].

\subsection{SUMMATION-CONCENTRATIONS}

Summation and use of Equation (14) yields

$$
\sum_{j=1}^{n}\left[S\left(X_{k}, \alpha_{j}\right)+S\left(X_{k}, \beta_{j}\right)\right]=\sum_{j=1}^{n}\left[S\left(X_{k}, \alpha_{j}\right)-S\left(X_{k}, \alpha_{j}\right)\right]=0 .
$$

In words, the percentage change in some dependent variable $X_{k}$ resulting from a one-percent change in the rate constant for a given net reaction or process, summed over all such reactions or processes in the system, must be zero.

This is a general constraint. When there are no branches in the system, and when the rate laws are independent of each other and linear functions of enzyme levels, then this generalized summation relationship reduces to the special case published by Heinrich and Rapoport [5]:

$$
\sum_{j=1}^{2 n} C_{E_{j}}^{X_{k}}=0
$$

where

$$
C_{l_{i}}^{X_{k}}=S\left(X_{k}, \alpha_{j}\right)\left[\text { or } S\left(X_{k}, \beta_{j}\right)\right] .
$$

'If there are branches in the flow of material and precursor-product constraints, but the rate laws still are independent and linear in enzyme concentration, then the corresponding relationships in BST and MCT are still equally valid. However, they involve different interpretations, as we shall see in Section 5. If the rate laws are not independent or not linear in enzyme concentration, then the relationships in MCT are no longer valid. 
154 MICHAEL A. SAVAGEAU, EBERHARD O. VOIT, AND DOUGLAS H. IRVINE

Note that in this case biosynthetic and degradative terms are not distinguished notationally, and thus $E$ is used as an analogy for both $\alpha$ and $\beta$.

\section{CONNFCTIVITY-CONCFNTRATIONS}

Substitution of the relationships in Equation (14) into Equation (16) yields

$$
\sum_{j=1}^{n} S\left(X_{i}, \beta_{j}\right) a_{j k}=\delta_{i k}
$$

or

$$
\sum_{j=1}^{n}\left[S\left(X_{i}, \beta_{j}\right) g_{j k}-S\left(X_{i}, \beta_{j}\right) h_{j k}\right]=\delta_{i k},
$$

since $a_{j k}=g_{j k}-h_{j k}$. Again using the relationships in Equation (14), one finds

$$
\sum_{j=1}^{n}\left[S\left(X_{i}, \alpha_{j}\right) g_{j k}+S\left(X_{i}, \beta_{j}\right) h_{j k}\right]=-\delta_{i k}
$$

In words, when the percentage change in some dependent variable $X_{i}$ resulting from a one-percent change in the rate constant for a given net reaction or process is multiplied by the kinetic order of that net reaction or process with respect to another dependent variable $X_{k}$ and these products are summed over all net reactions or processes in the system, the result is equal to zero if $i \neq k$ or minus one if $i=k$.

These results are true in general. When there are no branches in the system, and when the rate laws are independent of each other and linear functions of enzyme levels, then this generalized connectivity relationship reduces to the special case published by Westerhoff and Chen [33] (see footnote 1):

$$
\sum_{j=1}^{2 n} C_{E_{j}}^{X_{i}} \varepsilon_{X_{k}}^{j}=-\delta_{i k}
$$

Note that because biosynthetic and degradative reactions are not distinguished notationally, $\varepsilon$ is used for both $g$ and $h$. 


\subsection{SUMMATION-FLUXES}

Historically, specific relationships of this type were among the first of the summation and connectivity relationships to be found $[8,5]$. The verification of these relationships in BST [21, Chapter 9] was accomplished by first establishing the concentration relationships in the previous two sections. The procedure is as follows.

An arbitrary flux, $V_{k}$, through the pool $X_{k}$ is given by

$$
V_{k}=\alpha_{k} \prod_{j=1}^{n} X_{j}^{g_{k j}}
$$

By definition

$$
\begin{aligned}
& S\left(V_{k}, \alpha_{i}\right)=\frac{\partial \log V_{k}}{\partial \log \alpha_{i}}=\delta_{i k}+\sum_{j=1}^{n} g_{k j} S\left(X_{j}, \alpha_{i}\right), \\
& S\left(V_{k}, \beta_{i}\right)=\frac{\partial \log V_{k}}{\partial \log \beta_{i}}=\sum_{j=1}^{n} g_{k j} S\left(X_{j}, \beta_{i}\right) .
\end{aligned}
$$

When these terms are summed,

$$
\begin{aligned}
\sum_{i=1}^{n}[ & \left.S\left(V_{k}, \alpha_{i}\right)+S\left(V_{k}, \beta_{i}\right)\right] \\
& =1+\sum_{i=1}^{n} \sum_{j=1}^{n} g_{k j}\left[S\left(X_{j}, \alpha_{i}\right)+S\left(X_{j}, \beta_{i}\right)\right]=1
\end{aligned}
$$

The percentage change in the given flux resulting from a one-percent change in the rate constant of a net reaction or process, summed over all such reactions or processes, must be unity.

This is a general result, which, when there are no branches in the system, and when the rate laws are independent of each other and linear functions of enzyme levels, reduces to the special case first published by Kacser and Burns [8] and Heinrich and Rapoport [5] (see footnote 1):

$$
\sum_{i=1}^{2 n} C_{E_{i}}^{J_{k}}=1 .
$$

4.4. CONNECTIVITY - FLUXES

Specific relationships of this type also were first published by Kacser and Burns [8], and again the verification in BST proceeded from the relationships 
in Sections 4.1 and 4.2. Thus,

$$
\begin{aligned}
& \sum_{i=1}^{n}\left[S\left(V_{k}, \alpha_{i}\right) g_{i m}+S\left(V_{k}, \beta_{i}\right) h_{i m}\right] \\
& \quad=\sum_{i=1}^{n}\left\{\left[\delta_{i k}+\sum_{j=1}^{n} g_{k j} S\left(X_{j}, \alpha_{i}\right)\right] g_{i m}+\left[\sum_{j=1}^{n} g_{k j} S\left(X_{j}, \beta_{i}\right)\right] h_{i m}\right\} \\
& =g_{k m}-\sum_{i=1}^{n} \sum_{j=1}^{n} g_{k j} S\left(X_{j}, \beta_{i}\right) a_{i m} \\
& =g_{k m}-\sum_{j=1}^{n} g_{k j}\left[\sum_{i=1}^{n} S\left(X_{j}, \beta_{i}\right) a_{i m}\right] \\
& \quad=g_{k m}-\sum_{j=1}^{n} g_{k j}\left[\delta_{j m}\right]=0 .
\end{aligned}
$$

When the percentage change in an arbitrary flux $V_{k}$ through the pool $X_{k}$ resulting from a one-percent change in the rate constant for a given net reaction or process is multiplied by the kinetic order of that reaction or process with respect to an arbitrary dependent variable $X_{m}$ and summed over all such reactions or processes, the result must be zero.

Again, this is a general relationship, which under appropriate circumstances reduces to the special case described by Kacser and Burns [8] (see footnote 1):

$$
\sum_{i=1}^{2 n} C_{E_{i}^{k}}^{J_{k}} \varepsilon_{X_{m}}^{i}=0
$$

The results in this section show that constraint relations similar to, but more general than, the specific summation and connectivity relationships first described in MCT are inherent to the structure of the Power-Law Formalism that underlies both BST and MCT. These relationships provide another interesting perspective on the Power-Law Formalism, but they have not added fundamentally to or extended the range of application for BST. The complete solution to the problem of relating systemic behavior to underlying molecular determinants in steady state was provided by BST without the explicit use of the relationships in this section [17]. ${ }^{2}$

\footnotetext{
${ }^{2}$ From our demonstration that summation and connectivity relationships are none other than the familiar orthogonality relationships of linear systems, it is clear that any attempt to "prove" these relationships by first solving a set of linear equations for the sensitivities [control coefficients] (e.g., see Kacser and Porteous [9] and references in their "Reply" following Savageau [24]) is a circular argument. The solution of such equations requires orthogonality (or summation and connectivity) relationships in the first place.
} 


\section{BRANCHED BIOSYNTHETIC PATHWAY - AN EXAMPLE}

Although unbranched biosynthetic pathways appear to be governed by a single pattern of regulation, which by several criteria for functional effectiveness represents an optimal pattern [18, 19], branched biosynthetic pathways exhibit a diversity of patterns of regulation that are poorly understood. A predominant pattern in enteric bacteria, the nested pattern of regulation, has been analyzed in some detail $[18 ; 21$, Chapter 12]. The results of this analysis led to the prediction of multifunctional enzyme complexes among key enzymes and to a clear rationale in terms of regulatory purpose. Experimental evidence in support of these predictions has been discussed elsewhere [21, Chapter 12]. These results also led to the prediction of a new regulatory interaction in a system that only partially fulfilled the requirements for the normal nested pattern of regulation [10].

\subsection{REPRESENTATION IN BST}

Figure 1 represents a simplified model of such a branched biosynthetic system. The distinguishing features of this model are the existence of precursor-product relationships, branches in the flow of material, and complex regulatory interactions. The equations that describe this system in BST are

$$
\begin{aligned}
& d X_{1} / d t=\alpha_{1} X_{0}^{g_{10}} X_{2}^{g_{12}} X_{3}^{g_{13}}-\beta_{1} X_{1}^{h_{11}} X_{2}^{h_{12}} X_{3}^{h_{13}}, \\
& d X_{2} / d t=\alpha_{2} X_{1}^{g_{21}} X_{2}^{g_{22}}-\beta_{2} X_{2}^{h_{22}}, \\
& d X_{3} / d t=\alpha_{3} X_{1}^{g_{31}} X_{3}^{g_{33}}-\beta_{3} X_{3}^{h_{33}} .
\end{aligned}
$$

The rate laws for the two processes degrading $X_{1}$ have been combined into a net rate law, and then the system has been described in the Power-Law Formalism. As noted earlier [27], this description provides the natural

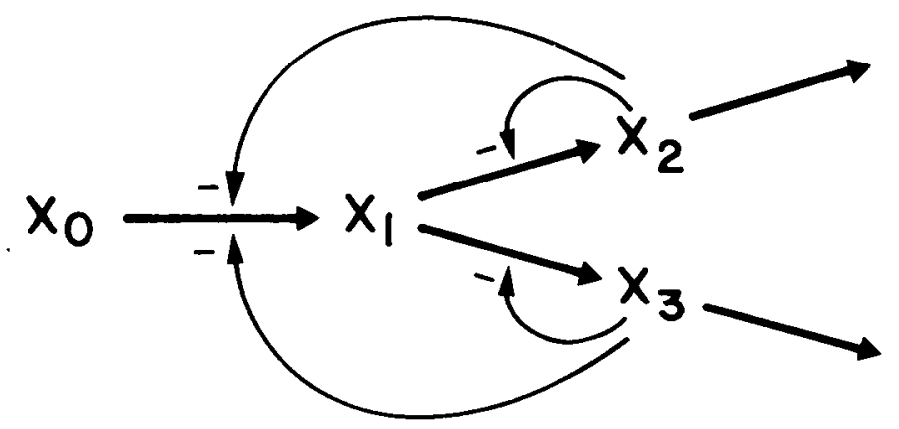

FIG. 1. Branched biosynthetic pathway. $X_{0}$ is an externally determined, independent concentration variable. The arrows from the dependent concentration variables $X_{2}$ and $X_{3}$ to the eenter of other arrows represent inhibitory interactions. 
parameters for expressing the steady-state existence $[14 ; 21$, Chapter 7] and local stability [20;21, Chapter 7] theorems, namely, the differences between the kinetic orders for net synthesis and net degradation $\left(g_{i j}-h_{i j}\right)$. It also makes transparent the relationships between the sensitivities with respect to the rate constants for synthetic and degradative reactions.

Although there are precursor-product relationships, one cannot equate parameters such as $h_{12}$ and $g_{22}$ or $h_{13}$ and $g_{33}$, because the net degradation of $X_{1}$ is not the same as the net synthesis of $X_{2}$ (or $X_{3}$ ). This is one reason why different symbols were originally introduced in BST $[14 ; 18 ; 21$, Chapter 12]. Nevertheless, the precursor-product constraints do lead to obvious relationships among these parameters. At steady-state operating points for this system

$$
\beta_{1} X_{10}^{h_{11}} X_{20}^{h_{12}} X_{30}^{h_{13}}=\alpha_{2} X_{10}^{g_{21}} X_{20}^{g_{22}}+\alpha_{3} X_{10}^{g_{31}} X_{30}^{g_{33}}
$$

and by definition of kinetic order $[14,17]$

$$
\begin{aligned}
& h_{11}=\frac{V_{2}}{V_{1}} g_{21}+\frac{V_{3}}{V_{1}} g_{31}, \\
& h_{12}=\frac{V_{2}}{V_{1}} g_{22}, \\
& h_{13}=\frac{V_{3}}{V_{1}} g_{33} ;
\end{aligned}
$$

also

$$
\beta_{1}=\left(V_{2}+V_{3}\right) X_{10}^{-h_{11}} X_{20}^{-h_{12}} X_{30}^{-h_{13}}
$$

where

$$
\begin{aligned}
& V_{2}=\alpha_{2} X_{10}^{g_{21}} X_{20}^{g_{22}}, \\
& V_{3}=\alpha_{3} X_{10}^{g_{31}} X_{30}^{g_{33}}, \\
& V_{1}=V_{2}+V_{3} .
\end{aligned}
$$

In words, the kinetic order with respect to a given $X_{i}$ in the net rate law is simply the sum of the corresponding kinetic orders in the individual rate laws, weighted according to the contributions of the individual fluxes to the net flux.

\subsection{EXPLICIT STEADY-STATE SOLUTION IN BST}

As in Section 2, the steady-state equations describing this system in logarithmic form can be written in a straightforward manner. If the determinant of the system matrix is nonzero, i.e.,

$$
|A|=-\left(h_{11} a_{22} a_{33}+g_{31} a_{22} a_{13}+g_{21} a_{33} a_{12}\right) \neq 0,
$$


then a steady-state solution exists and can be written explicitly [cf. Equation (3)]:

$$
\begin{aligned}
& \left.\begin{array}{l}
y_{1} \\
y_{2} \\
y_{3}
\end{array}\right]=\frac{1}{|A|}\left[\begin{array}{r}
-g_{10} a_{22} a_{33} \\
g_{10} g_{21} a_{33} \\
g_{10} g_{31} a_{22}
\end{array}\right] y_{0} \\
& \left.+\frac{1}{|A|}\left[\begin{array}{ccc}
a_{22} a_{33} & -a_{12} a_{33} & -a_{13} a_{22} \\
-g_{21} a_{33} & -\left(h_{11} a_{33}+g_{31} a_{13}\right) & g_{21} a_{13} \\
-g_{31} a_{22} & g_{31} a_{12} & -\left(h_{11} a_{22}+g_{21} a_{12}\right)
\end{array}\right] \begin{array}{l}
b_{1} \\
b_{2} \\
b_{3}
\end{array}\right] .
\end{aligned}
$$

Thus, the parameter values and the value of the independent concentration variable $X_{0}$ determine the nonzero steady-state values of the dependent concentration variables $X_{1}, X_{2}$, and $X_{3}$. From these equations one can calculate directly (or see by inspection) the change in dependent concentrations that would result from a change in independent concentration $\left(L_{i v}\right)$, a change in kinetic order $\left[S\left(y_{l}, h_{i k}\right)\right]$, or a change in rate constant $\left[S\left(X_{i}, \beta_{j}\right)\right]$.

As in Section 3, the systemic properties (logarithmic gains and sensitivities) can be calculated directly and expressed in terms of the underlying molecular parameters ( $\alpha$ 's, $\beta$ 's, $g$ 's, and $h$ 's). One also can invert these relationships and express the underlying molecular parameters in terms of the systemic properties, although in some cases this calculation requires additional information about changes in fluxes. In this example

$$
\begin{aligned}
& h_{11}=\frac{M_{23} M_{32}-M_{22} M_{33}}{|M|}, \\
& g_{21}=\frac{M_{23} M_{31}-M_{21} M_{33}}{|M|}, \\
& g_{31}=\frac{M_{21} M_{32}-M_{22} M_{31}}{|M|}, \\
& g_{10}=-\frac{L_{30}}{M_{31}}, \\
& a_{12}=\frac{M_{13} M_{32}-M_{12} M_{33}}{|M|}, \\
& a_{22}=\frac{M_{11} M_{33}-M_{13} M_{31}}{|M|}, \\
& a_{13}=\frac{M_{12} M_{23}-M_{13} M_{22}}{|M|}, \\
& a_{33}=\frac{M_{11} M_{22}-M_{12} M_{21}}{|M|},
\end{aligned}
$$


where

$$
\begin{aligned}
|M|= & M_{11} M_{22} M_{33}+M_{12} M_{23} M_{31}+M_{13} M_{32} M_{21} \\
& -M_{11} M_{23} M_{32}-M_{22} M_{13} M_{31}-M_{33} M_{12} M_{21} .
\end{aligned}
$$

The first four kinetic orders in Equation (38) are uniquely related to the systemic properties; the remaining eight are not related uniquely but in pairs $\left(g_{i,}-h_{i j}\right)$ to the systemic properties. Measurements of changes in steadystate fluxes can be used to obtain the individual molecular parameters. For example, the parameters $\beta_{3}$ and $h_{33}$ in Equation (27) can be obtained from measurements of $V_{3}$ and $X_{3}$ by linear regression according to the formula

$$
\log V_{3}=\log \beta_{3}+h_{33} \log X_{3}
$$

The conditions governing local dynamic stability require that

$$
|A|=-\left(h_{11} a_{22} a_{33}+g_{31} a_{22} a_{13}+g_{21} a_{33} a_{12}\right)<0
$$

for the system to be stable $[18 ; 20 ; 21$, Chapter 7]. The local dynamic behavior of the system follows from Equation (27) and has been explored elsewhere [21, Chapter 12]. As we shall see in Section 5.6, the condition in Equation (40) also is essential for interpreting the direction of change in variables as the system moves from one steady state to another.

\subsection{SUMMATION AND CONNECTIVITY RELATIONSHIPS IN BST}

One easily can verify that all the summation and connectivity relations are satisfied. For example, the concentration summation for $X_{2}$ is given by

$$
\begin{aligned}
& S\left(X_{2}, \alpha_{1}\right)+S\left(X_{2}, \beta_{1}\right) \\
& \quad+S\left(X_{2}, \alpha_{2}\right)+S\left(X_{2}, \beta_{2}\right)+S\left(X_{2}, \alpha_{3}\right)+S\left(X_{2}, \beta_{3}\right)=0
\end{aligned}
$$

or

$$
\begin{gathered}
g_{21} a_{33}-g_{21} a_{33}+\left(h_{11} a_{33}+g_{31} a_{13}\right)-\left(h_{11} a_{33}+g_{31} a_{13}\right) \\
-g_{21} a_{13}+g_{21} a_{13}=0
\end{gathered}
$$

by inspection of Equation (37). Note that the determinant $|A|$ common to each sensitivity has been canceled for simplicity. Because the sensitivities with respect to net synthesis and net degradation are always equal in magnitude but opposite in sign, this is satisfied trivially. However, the precursor-product constraints prevent one from physically altering the parameters $\alpha_{2}$ or $\alpha_{3}$ without concomitantly changing the parameter $\beta_{1}$. Thus, the second sensitivity in Equation (41) actually is composed of two components; the sum of one of these and the third sensitivity in Equation (41) will represent the sensitivity of $X_{2}$ with respect to the physical change in 
$\alpha_{2}$, while the sum of the remaining component and the fifth sensitivity in Equation (41) will represent the sensitivity of $X_{2}$ with respect to the physical change in $\alpha_{3}$. Again, these individual contributions are clearly distinguished mathematically even though they cannot be distinguished physically. We can see these relationships explicitly if we express this summation relationship after imposing the precursor-product constraints [Equations (32)-(34)] and making the appropriate calculations:

$$
S\left(X_{2}, \alpha_{1}\right)+S^{\prime}\left(X_{2}, \alpha_{2}\right)+S\left(X_{2}, \beta_{2}\right)+S^{\prime}\left(X_{2}, \alpha_{3}\right)+S\left(X_{2}, \beta_{3}\right)=0
$$

or

$$
\begin{aligned}
g_{21} a_{33}+ & {\left[\left(h_{11} a_{33}+g_{31} a_{13}\right)-\frac{V_{2}}{V_{1}} g_{21} a_{33}\right]-\left(h_{11} a_{33}+g_{31} a_{13}\right) } \\
& -\left[g_{21} a_{13}+\frac{V_{3}}{V_{1}} g_{21} a_{33}\right]+g_{21} a_{13}=0,
\end{aligned}
$$

where again $|A|$ has been canceled for convenience.

Comparison of Equations (41) and (42) shows that

$$
\begin{aligned}
& S^{\prime}\left(X_{2}, \alpha_{2}\right)=S\left(X_{2}, \alpha_{2}\right)+\frac{V_{2}}{V_{1}} S\left(X_{2}, \beta_{1}\right), \\
& S^{\prime}\left(X_{2}, \alpha_{3}\right)=S\left(X_{2}, \alpha_{3}\right)+\frac{V_{3}}{V_{1}} S\left(X_{2}, \beta_{1}\right) .
\end{aligned}
$$

The terms in the brackets in Equation (42) represent the sensitivity to a physical change in $\alpha_{2}$ and $\alpha_{3}$. Within each bracket one can identify the individual contributions from the effects on synthesis and degradation, even though these individual effects cannot be physically separated. The branching in this example implies that the synthesis of each end product is only equivalent to the relevant fraction of the degradation of the branchpoint metabolite $X_{1}$. Thus, the two degradative contributions are weighted according to the fraction of the total flux in each branch.

Other summation and connectivity relations could be examined in the same fashion.

\subsection{REPRESENTATION IN MCT}

As we have seen, the general solution in BST [17] applies directly to systems with branches. For these systems, MCT reached essentially the same solution when Heinrich and Rapoport [6] provided the additional relationships needed in MCT to deal with branches:

$$
\alpha_{X_{s} r} V_{r}+\sum_{i=1}^{n} \sum_{k=1}^{m} \alpha_{X_{s} i} V_{i} \varepsilon_{X_{k}}^{i} C_{E_{r}}^{X_{k}}=0
$$


and

$$
J C_{E_{r}}^{J}=\sum_{i=1}^{n} \sum_{k=1}^{m} \beta_{J i} V_{i} \varepsilon_{X_{k}^{i}}^{i} C_{E_{r}}^{X_{k}}+\beta_{J r} V_{r} .
$$

The calculation of a typical sensitivity (control coefficient) in MCT proceeds in a very different fashion from that illustrated above for BST. In MCT the branched system in Figure 1 is described by the following equations (see $[27])$ :

$$
\begin{aligned}
& d X_{1} / d t=E_{1} X_{0}^{e^{1} X_{0}} X_{2}^{e^{1} X_{2}} X_{3}^{e^{1} X_{3}}-E_{4} X_{1}^{\epsilon^{4} X_{1}} X_{2}^{t^{4} X_{2}}-E_{5} X_{1}^{\tau^{5} x_{1}} X_{3}^{\varepsilon^{5} X_{3}}, \\
& d X_{2} / d t=E_{4} X_{1}^{\varepsilon^{4} X_{1}} X_{2}^{\varepsilon^{4} X_{2}}-E_{2} X_{2}^{\varepsilon_{x}^{2}}, \\
& d X_{3} / d t=E_{5} X_{1}^{\varepsilon_{X_{1}}} X_{3}^{\varepsilon_{X_{3}}^{5}}-E_{3} X_{3}^{\varepsilon_{X_{3}}^{3}},
\end{aligned}
$$

the fundamental difference being the application of the Power-Law Formalism before summing the individual rates of degradation for $X_{1}$ [cf. Equation (27)]. In this MCT form there is no general or symbolic steady-state solution that can be obtained explicitly. Consequently there are no simple steady-state existence and local stability theorems corresponding to Equations (36) and (40) that can be stated explicitly in terms of kinetic orders and rate constants.

\subsection{SUMMATION AND CONNECTIVITY RELATIONSHIPS IN MCT}

Relationships between elemental component and system behavior in MCT are obtained by use of the summation and connectivity relationships. In general there are $n$ summation relationships [Equation (18)] and $n \times n$ connectivity relationships [Equation (20)] for $n$ concentration variables, and $N$ summation relationships [Equation (24)] and $n \times N$ connectivity relationships [Equation (26)] for $N$ flux variables. In examples such as this, which involve branches in the flow of material, one also must use the auxiliary relationships developed by Heinrich and Rapoport [6]. In general there are $n \times N$ relating concentration variables [Equation (45)] and $N \times N$ relating flux variables [Equation (46)]. The resulting set of equations is highly redundant, and one must find a linearly independent set (e.g., see [1]) if one is to obtain a unique solution.

As an illustration let us relate the systemic property $C_{L_{3}}^{X_{2}}$ to the elasticities and fluxes of the system in steady state. In this case there are 72 equations specified by Equations (18), (20), (24), (26), (45), and (46). We will not go through the details of determining a linearly independent set that will allow 
one to solve uniquely for $C_{E_{3}}^{X_{2}}$, but simply indicate that the following three equations will suffice.

When $s=1$ and $r=3$, Equation (45) implies

$$
-\left(V_{4} \varepsilon_{X_{1}}^{4}+V_{5} \varepsilon_{X_{1}}^{5}\right) C_{L_{3}}^{X_{1}}+\left(V_{1} \varepsilon_{X_{2}}^{1}-V_{4} \varepsilon_{X_{2}}^{4}\right) C_{E_{3}}^{X_{2}}+\left(V_{1} \varepsilon_{X_{3}}^{1}-V_{5} \varepsilon_{X_{3}}^{5}\right) C_{L_{3}}^{X_{3}}=0 .
$$

Similarly, when $s=2$ and $r=3$,

$$
V_{4} \varepsilon_{X_{1}}^{4} C_{E_{3}}^{X_{1}}+\left(V_{4} \varepsilon_{X_{2}}^{4}-V_{2} \varepsilon_{X_{2}}^{2}\right) C_{E_{3}}^{X_{2}}=0,
$$

and when $s=3$ and $r=3$,

$$
-V_{3}+V_{5} \varepsilon_{X_{1}}^{5} C_{E_{3}}^{X_{1}}+\left(V_{5} \varepsilon_{X_{3}}^{5}-V_{3} \varepsilon_{X_{3}}^{3}\right) C_{E_{3}}^{X_{3}}=0 .
$$

These represent three linear equations in the three control coefficients, and thus can be solved under appropriate conditions for the coefficient of interest:

$$
\begin{aligned}
C_{Z_{3}}^{X_{2}}=- & {\left[\varepsilon_{X_{1}}^{4}\left(\varepsilon_{X_{3}}^{1}-\frac{V_{5}}{V_{1}} \varepsilon_{X_{3}}^{5}\right)\right] } \\
\times & {\left[\left(\frac{V_{4}}{V_{1}} \varepsilon_{X_{1}}^{4}+\frac{V_{5}}{V_{1}} \varepsilon_{X_{1}}^{5}\right)\left(\varepsilon_{X_{2}}^{4}-\varepsilon_{X_{2}}^{2}\right)\left(\varepsilon_{X_{3}}^{5}-\varepsilon_{X_{3}}^{3}\right)\right.} \\
& +\varepsilon_{X_{1}}^{5}\left(\varepsilon_{X_{2}}^{4}-\varepsilon_{X_{2}}^{2}\right)\left(\varepsilon_{X_{3}}^{1}-\frac{V_{5}}{V_{1}} \varepsilon_{X_{3}}^{5}\right) \\
& \left.+\varepsilon_{X_{1}}^{4}\left(\varepsilon_{X_{3}}^{5}-\varepsilon_{X_{3}}^{3}\right)\left(\varepsilon_{X_{2}}^{1}-\frac{V_{4}}{V_{1}} \varepsilon_{X_{2}}^{4}\right)\right]^{-1}
\end{aligned}
$$

One would have to go through a similar process, although perhaps involving a different set of equations, for each coefficient of interest.

Fell and Sauro [4] bave described a simpler alternative for generating a set of linearly independent equations in MCT; the same results are obtained by either method.

\subsection{COMPARISON OF RESULTS FROM MCT AND BST}

The results in Section 5.5, although expressed in symbolic form, do not allow one to draw general conclusions, such as the direction of change in $X_{2}$ following an increase in $E_{3}$, that are independent of the particular parameter values. This is because the sign of the denominator in Equation (51) is indeterminate. 
One can compare this result in MCT with the corresponding result in BST by noting the following relationships between their symbols:

$$
\begin{aligned}
& h_{11}=\frac{V_{4}}{V_{1}} \varepsilon_{X_{1}}^{4}+\frac{V_{5}}{V_{1}} \varepsilon_{X_{1}}^{5}, \\
& h_{12}=\frac{V_{4}}{V_{1}} \varepsilon_{X_{2}}^{4}, \\
& h_{13}=\frac{V_{5}}{V_{1}} \varepsilon_{X_{3}}^{5} .
\end{aligned}
$$

The other elasticities can be translated directly into the corresponding kinetic orders, and Equation (51) reduces to

$$
S\left(X_{3}, \beta_{3}\right)=-\frac{g_{21} a_{13}}{h_{11} a_{22} a_{33}+g_{31} a_{22} a_{13}+g_{21} a_{33} a_{12}},
$$

which could have been obtained simply by inspection of Equation (37).

Although the alternative expressions in Equations (51) and (53) are mathematically equivalent, the attainment, biological meaning, and interpretation of Equation (53) are more transparent. All sensitivities with respect to changes in enzyme levels [e.g., Equation (53)] can be obtained directly from the general symbolic solutions in Equation (37). Equation (53) illustrates the fundamental importance of the differences between kinetic orders for net synthesis and net degradation, which are made explicit in BST. In this example, the relative magnitudes of $g_{13}$ and $h_{13}$ (in $a_{13}=g_{13}-h_{13}$ ) determine completely the direction of change in $X_{2}$. An increase in the amount of the enzyme degrading $X_{3}$ causcs a change in the steady-state value of the other product $X_{2}$. If the system is stable, then the denominator in Equation (53) is positive (regardless of the particular values of the parameters). The parameter $g_{21}$ is positive, so $X_{2}$ will either increase or decrease depending upon the sign of the parameter $a_{13}$. If $a_{13}<0\left(g_{13}<h_{13}\right)$, then $X_{2}$ will increase; if $a_{13}>0\left(g_{13}>h_{13}\right)$, then $X_{2}$ will decrease. Without the stability condition [Equation (40)] to show that the denominator is positive, one would have no way to predict the direction of change in $X_{2}$.

This example illustrates the analysis of a general class of biochemical systems and shows that one can make general predictions that are independent of the particular numerical values for the specific parameters. It also shows clearly the advantages of aggregating rate laws for net synthesis and net degradation (see also [32]), and the importance of the stability relationships $[18 ; 20 ; 21$, Chapter 12$]$ for interpreting steady-state results.

\section{DISCUSSION}

We began the previous paper by noting that the original presentation and subsequent development of MCT have not acknowledged the earlier 
results in BST nor shown how these have been augmented in MCT [27]. A possible explanation is that MCT and BST were seen as unrelated because of differences in the level of formal development and in the existence of specific summation and connectivity relationships. This impression persisted because MCT had never been explicitly expressed in terms of the fundamental, underlying mathematical structure and because it had never been systematically compared with the earlier BST.

The results in the previous paper demonstrated that in fact the above differences are rather superficial and that at a fundamental level the logical content of MCT is a subset of that of BST. BST is a more comprehensive theory, has a more systematic structure based explicitly on the underlying formalism, and appears to have a broader range of valid application [32].

Perhaps the major stumbling block to recognizing MCT's relatedness to BST is the summation and connectivity relationships. These are the most visible and central feature of MCT, while in BST they appear to be invisible and peripheral.

In this paper we have claborated on the generalized summation and connectivity relationships in BST that were used to verify [21, Chapter 9] the specific relationships described by Kacser and Burns [8] and Heinrich and Rapoport [5]. It is demonstrated that these generalized relationships are a natural part of BST. They are equivalent to the orthogonality properties, which are inherent to the steady-state equations in the Power-Law Formalism because of their linear structure, and they are expressed in terms of the fundamental parameters: rate constants and kinetic orders. In contrast, special versions of such relationships were presented in an ad hoc fashion and then made the basis of MCT $[8,5,9]$. These specific relationships were expressed in terms of kinetic orders (elasticities) and enzyme levels or molecular activities. The latter are not fundamental parameters in the underlying formalism, and the resulting relationships are valid only for systems in which the rate laws for the individual enzymes are independent of each other and are linear functions of enzyme concentration and molecular activity. As we have seen, BST and MCT do not differ in that one has summation and connectivity relationships while the other does not; these types of relationships exist in both approaches. Nevertheless, these relationships play significantly different roles in BST and MCT.

As pointed out in this paper, summation and connectivity relationships have no operational role in BST. They are only corollaries in BST, and need never be made manifest during the development of the theory or for purposes of application. In this sense they play no significant role. One can do everything directly in BST and never explicitly invoke the summation or connectivity relationships. For this reason we have never thought it important to highlight these relationships, except to note that they can be verified readily in BST [21, Chapter 9]. 
In contrast, summation and connectivity relationships have held a special place in MCT. Two distinct roles can be identified: they serve as "conservation laws," and they serve as the "primitive equations" for relating systemic and molecular properties, and thus as the port of entry into the theoretical domain it shares with BST.

The original development and use of the summation and connectivity relationships within MCT occurred in the context of sequential chains of simple enzymatic reactions [8, 5], and this gave rise to the notion of sensitivities (control coefficients) as positive fractional quantities that must sum to one for the flux in the system. Sensitivity ("control") is distributed or shared among all the enzymes of the system. If one enzyme has a sensitivity (control coefficient) of one, then it would be "controlling" and all others would have values of zero. There is a "unit" amount of "control" and it is conserved. If the value goes up in one part of the system, then it must go down elsewhere. In this sense these quantities might be considered analogous to energy, and the summation relationships to the conservation of energy.

One might use such a "conservation law" to test the validity of a model. If the experimentally determined sensitivities (control coefficients) for each of the processes in the model do not add up to one, then the model is lacking some important element. If they do, then the model is complete. However, this role for summation relationships can be criticized on several levels.

First, this role can only be fulfilled within the limited context of sequential chains of simple enzymatic reactions, and then only if one tabulates experimentally determined values (e.g. see [30]). In cases where the values are calculated from the model (e.g., see [12]) the test is no longer valid, since, as noted earlier, these relationships always will sum to unity-regardless of whether the model is valid or invalid. It is only the discrepancy between experimental measurements and unity (the model result) that is of diagnostic value.

Second, sensitivities (control coefficients) need not have positive fractional values when more complex systems, such as those containing enzyme cascades $[17 ; 21$, Chapter $13,15,16]$ or branches in the flow of material $[18 ; 21$, Chapter 12], are considered. When sensitivities (control coefficients) can be negative as well as positive and when they can have magnitudes greater than unity, the ability to test for completeness of a model is lost. Even if experimentally determined values sum to unity, one might have omitted several important elements from the model; positive and negative contributions from neglected elements can cancel fortuitously. There can be several enzymes with sensitivities (control coefficients) greater than or equal to unity, and others need not have values equal to zero. All that remains is the individual sensitivities, and the "conservation laws" do not allow one to deduce directly the relationship among their values, as they do for simpler systems. 
Third, the summation and connectivity relationships in MCT are expressed in terms of enzyme activities, enzyme levels, or molecular activities $[8,5,9]$; these are not valid except for simple systems in which the rate laws are independent of each other and proportional to enzyme concentration or molecular activity $[24,27]$. Thus, in more general cases the idea of "conservation law" is meaningless.

The more fundamental role of the summation and connectivity relationships in MCT is that of "primitive equations" used to obtain the relationship between systemic and molecular properties in biochemical systems. This role also is subject to several levels of criticism. First, these summation and connectivity relationships are not sufficient to obtain the explicit relationships between systemic and molecular parameters, except in the special case of sequential chains of simple enzymatic reactions $[8,5]$. One has to introduce auxiliary relationships [6] in the case of branching systems in order to obtain in MCT a solution comparable to that obtained earlier in BST $[14,17]$. Second, the summation and connectivity relationships in MCT do not give conditions for the existence or the local stability of a steady state. As the basis for a general theory, these summation and connectivity relationships in MCT are therefore inferior to the underlying equations of BST, which do yield such conditions $[14,20]$. Third, and most fundamental, these summation and connectivity relationships are based on rate laws that are assumed to be independent of each other and linearly related to enzyme level and molecular activity [8, 5, 9]. Since biological systems are known to contain reactions that do not fit this simplifying assumption [24, 27], these summation and connectivity relationships are not generally valid. They do have an intuitive appeal if they are accepted uncritically, which may be fostered by claims [9] that they are entirely general. To the best of our knowledge, the conditions identified here, which are required for the validity of the summation and connectivity relationships in MCT, have never been verified in any application.

From these results one can conclude that MCT is not a new theory unrelated to BST. In fact, MCT is but a special case or subset of BST. By attempting to make specific summation and connectivity relationships the basis of a theory (MCT) one is prevented from recognizing the explicit structure of the underlying formalism. Paradoxically, one also is prevented from recognizing the more general expression and interpretation of summation and connectivity relationships themselves. As we have seen, the rate-constant parameters that are essential for the more general expression of these relationships are not present in MCT. The sensitivities with respect to the most numerous parameters in a system - the kinetic orders-also are absent from MCT.

Finally, it should be pointed out that BST, by focusing upon the explicit structure of the underlying formalism, has led to a natural extension of the 
theory from approximate descriptions and local ranges of valid application to "exact" descriptions and global ranges of valid application. This extension of the theory is beyond the scope of this paper and is published elsewhere (for a review see Savageau and Voit [26]).

This work was supported in part by U.S. Public Health Service Grant $G M-30054$ from the National Institute of Health. The material presented in this paper was part of a larger manuscript circulated among Drs. $R$. Heinrich, $H$. Kacser, J. Porteous, S. Rapoport, and H. Westerhoff in March 1985. We thank them for their useful comments.

\section{REFERENCES}

1 R. Bellman, Introduction to Matrix Analysis, McGraw-Hill, New York, 1960.

2 H. W. Bode, Network Analysis and Feedback Amplifier Design, Van Nostrand, Princeton, N. J., 1945.

3 J. B. Cruz (Ed.), System Sensitivity Analysis, Dowden, Hitchinson and Ross, Stroudsburg, Pa. 1973.

4 D. A. Fell and H. M. Sauro, Metabolic control and its analysis, European J. Biochem. 148:555-561 (1985).

5 R. Heinrich and T. A. Rapoport, A linear steady-state treatment of enzymatic chains: General properties, control and effector strength, European, J. Biochem. 42:89-95 (1974).

6 R. Heinrich and T. A. Rapoport, Mathematical analysis of multienzyme systems 2. Steady-state and transient control, Biosystems 7:130-136 (1975).

7 D. H. Irvine and M. A. Savageau, Network regulation of the immune response: Alternative control points for suppressor modulation of effector lymphocytes, $J$. Immunol. 134:2100-2116 (1985).

8 H. Kacser and J. A. Burns, The control of flux, Symp. Soc. Exp. Biol. 27:65-104 (1973).

9 H. Kacser and J. W. Porteous, Control of metabolism: What do we have to measure?, Trends Biochem. Sci. 12:5-14 (1987).

10 A. M. Kotre, S. J. Sullivan, and M. A. Savageau, Metabolic regulation by homoserine in Escherichia coli B/r, J. Bacteriol. 116:663-672 (1973).

11 S. J. Mason, Feedback theory-some properties of signal flow graphs, Proc. I.R.E. 41:1144-1156 (1953).

12 T. A. Rapoport, R. Heinrich, and S. M. Rapoport, The regulatory principles of glycolysis in erythrocytes in vivo and in vitro, Biochem. J. 154:449-469 (1976).

13 M. A. Savageau, Biochemical systems analysis. I. Some mathematical properties of the rate law for the component enzymatic reactions, J. Theoret. Biol. 25:365-369 (1969).

14 M. A. Savageau, Biochemical systems analysis. II. The steady-state solutions for an $n$-pool system using a power-law approximation, J. Theoret. Biol. 25:370-379 (1969).

15 M. A. Savageau, Biochemical systems analysis. III. Dynamic solutions using a powerlaw approximation, J. Theoret. Biol. 26:215-226 (1970).

16 M. A. Savageau, Parameter sensitivity as a criterion for evaluating and comparing the performance of biochemical systems, Nature 229:542-544 (1971).

17 M. A. Savageau, Concepts relating the behavior of biochemical systems to their underlying molccular propertics, Arch. Biochem. Biophys. 145:612-621 (1971). 
18 M. A. Savageau, The behavior of intact biochemical control systems, Curr. Top. Cell. Reg. 6:63-130 (1972).

19 M. ^. Savageau, Optimal design of feedback control by inhibition: Steady-state considerations, J. Mol. Evol. 4:139-156 (1974).

20 M. A. Savageau, Optimal design of feedback control by inhibition: Dynamic considerations, J. Mol. Evol. 5:199-222 (1975).

21 M. A. Savageau, Biochemical Systems Analysis: A Study of Function and Design in Molecular Biology, Addison-Wesley, Reading, Mass., 1976.

22 M. A Savageau, Models of gene function: General methods of kinetic analysis and specific ecological correlates, in Foundations of Biochemical Engineering: Kinetics and Thermodynamics in Biological Systems (H. W. Blanch, E. T. Papoutsakis, and G. Stephanopoulos, Eds.), Amer. Chem. Soc., Washington, 1983, pp. 3-25.

23 M. A. Savageau, A theory of alternative designs for biochemical control systems, Biomed. Biochim. Acta 44:875-880 (1985).

24 M. A. Savageau, Control of metabolism: Where is the theory?, Trends Biochem. Sci. $12: 219-220(1987)$.

25 M. A. Savageau and D. A. Lapointe, Optimization of kinetic proofreading: A general method for derivation of the constraint relations and an exploration of a specific case, J. Theoret. Biol. 93:157-177 (1981).

26 M. A. Savageau and E. O. Voit, Recasting nonlinear differential equations as S-systems: A canonical nonlinear form, Math. Biosci., to appear.

27 M. A. Savageau, E. O. Voit, and D. H. Irvine, Biochemical systems theory and metabolic control theory. 1. Fundamental similarities and differences, Math. Biosci., 86:127-145 (1987).

28 A. Sorribas, Discovering another view of control analysis, Trends Biochem. Sci. 12:221-222 (1987).

29 J. G. Truxal, Automatic Feedback Control Systems Synthesis, McGraw Hill, New York, 1955

30 A. J. Verhoeven. P. Kamer, A. K. Groen, and J. M. Tager, Effects of thyroid hormone on mitrochondrial oxidative phosphorylation, Biochem. J. 226:183-192 (1985).

31 E. O. Voit, Control in perspective, Trends Biochem. Sci. 12:221 (1987).

32 E. O. Voit and M. A. Savageau, Accuracy of alternative representations for integrated biochemical systems, Biochemistry, in press.

33 H. V. Westerhoff, and Y.-D. Chen, How do enzyme activities control metabolite concentrations?, European J. Biochem. 142:425-430 (1984). 